like framework displaying a new hexagonal-packed net topology. All Lu-ATA isomeric phases are porous and display different gas-uptake behaviour towards carbon dioxide as a function of polymeric network arrangement. The luminescent properties of Lu-ATA frameworks in solid state as well as in suspension in the presence of different solvents reveal a solvent dependent emission.

References:

11] Aakeroy, C. B. et al. (2010). CrystEngComm, 12, 22-43.

[2] Jeong, S. et al. (2014). Chem. Mater., 26, 1711-1719.

[3] Konstas, K. et al. (2014). CrystEngComm, 16, 8937-8940.

Keywords: metal-organic frameworks, supramolecular isomerism, luminescence
MS32-P07

\section{Nanosheets of non-layered aluminium metal-organic frameworks through a surfactant-assisted method}

Alexey Pustovarenko ${ }^{1}$, Maarten G. Goesten ${ }^{2}$, Sumit Sachdeva ${ }^{3}$, Zakariae Amghouz ${ }^{4}$, Alla Dikhtiarenko ${ }^{1}$, Ernst J. R. Sudhölter ${ }^{3}$, Freek Kapteijn ${ }^{3}$, Beatriz Seoane ${ }^{5}$, Jorge Gascon ${ }^{1}$

1. Kaust Catalysis Center, King Abdullah University of Science and Technology, Thuwal, Saudi Arabia

2. Department of Chemistry and Chemical Biology, Cornell University, Cornell, United States of America

3. Department of Chemical Engineering, Delft University of Technology, Delft, Netherlands (Holland, Europe)

4. Department of Materials Science and Metallurgical Engineering, Polytechnic School of Engineering, University of Oviedo, Gijón, Spain

5. Debye Institute for Nanomaterials Science, Utrecht University, Utrecht, Netherlands (Holland, Europe)

email: oleksii.pustovarenko@kaust.edu.sa

During the last decade, the synthesis and application of metal-organic framework (MOF) nanosheets has received growing interest, ${ }^{[1]}$ showing unique performances for different technological applications. ${ }^{[2]}$ Despite the advances reported, the applicability of the synthetic methods developed so far is mainly restricted to MOFs possessing a layered structure. These MOFs consist of stacked 2D layers, being more prone to the formation of nanosheets. However, this subfamily of compounds only embodies a small fraction of the MOF structures reported to date, severely limiting the potential of this promising type of $2 \mathrm{D}$ nanomaterials. To the best of our knowledge, only one very recent example has been reported so far on the top-down exfoliation of 3D MOFs. ${ }^{[3]}$ However, top-down approaches are often associated with particle fragmentation and re-aggregation of the detached sheets. A bottom-up approach for the synthesis of 3D MOF has never been reported.

Within this context, we present a bottom-up surfactant-assisted synthetic approach for the fabrication of free-standing nanosheets of various non-layered 3D Al-containing MOFs, broadening the scope of MOF nanosheets application. Surfactant-assisted pre-organization of the metallic precursor prior to MOF synthesis allows for the manufacture of non-layered Al-containing MOF lamellae. These MOF nanosheets are shown to exhibit a superior performance over other crystal morphologies for both chemical sensing and gas separation. Gas separation behaviour of MOF nanosheets embedded in polymer matrix was explored for $\mathrm{CO}_{2} / \mathrm{CH}_{4}$ mixture and compared to the nanoparticle-containing composite. The $\mathrm{NH}_{2}-\mathrm{MIL}-53(\mathrm{Al})$ nanolamellae/ Matrimid ${ }^{\circledR}$ mixed matrix membrane showed a 10-fold increase in $\mathrm{CO}_{2}$ permeability along with a doubled selectivity in the separation of $\mathrm{CO}_{2} / \mathrm{CH}_{4}$ mixtures. Moreover, sensing studies on $\mathrm{NH}_{2}-\mathrm{MIL}-53(\mathrm{Al})$ nanolamellae-coated device suggested the enhanced sensing performance towards different types of alcohols. As revealed by electron microscopy and diffraction, this superior performance arises from the shorter diffusion pathway in MOF nanosheets, whose one-dimensional channels are oriented along the shortest particle dimension. 
References:

[1] Amo-Ochoa, P. et al. (2010). Chem. Commun., 46, 3262.

[2] Peng, Y. et al. (2014). Science, 346, 1356.

[3] Cliffe, M. J. et al. (2017). J. Am. Chem. Soc., 139, 5397.

Keywords: MOF nanolamellae, chemical sensing, molecular recognition

\section{MS32-P08}

\section{Pharmaceutical salts of antidiabetic drugs: mechanochemical synthesis, solid state characterization and solubility evaluation}

Duane Choquesillo-Lazarte ${ }^{1}$, Cristóbal Verdugo-Escamilla ${ }^{1}$, Argoitz Calvo-Borika², Raquel Fernández-Penas ${ }^{1}$, Antonio RodríguezDiéguez ${ }^{2}$

1. Laboratorio de Estudios Cristalográficos, IACT, CSIC-UGR, Granada, Spain

2. Departamento de Química Inorgánica, Facultad de Ciencias, UGR, Granada, Spain

email: duane.choquesillo@csic.es

The interest in multicomponent solid forms has increased in the last years within the pharmaceutical industry and also the solid-state community due to the possibility of obtaining materials with improved properties ${ }^{1,2}$. Crystallization strategies, supported by solvent- and solid-based ${ }^{3}$ techniques, have also received attention in the search and development of efficient methodologies for the screening of multicomponent crystals.

In this work, two antidiabetic drugs with limited aqueous solubility, chlorpropamide and tolbutamide, were selected to develop multicomponent forms on the basis of the synthon types using a series of coformers. Liquid Assisted Grinding (LAG) was used as a mechanochemical synthetic tool. Attempts to produce salts by LAG led to the formation of polycrystalline material. These solids were then characterized by powder X-ray diffraction as well as by spectroscopic and thermal methods. Recrystallization by slow solvent evaporation was carried out when the above-referred techniques strongly suggest the formation of a new solid form. In those cases where suitable crystals were obtained, single crystal $\mathrm{X}$-ray diffraction experiments were performed. Solubility determination of the selected solid forms has proved the advantage they offer over their corresponding parent APIs.

References:

[1] N. Blagden, D.J. Berry, A. Parkin, A., H. Javed, A. Ibrahim, P.T. Gavan, L.L. De Matos, C.C. Seaton. New J. Chem. 2008, 32, 1659-1672.

[2] D.J. Berry, J.W. Steed. Advanced Drug Delivery Reviews . 2017, $117,3-24$,

[3] J.-L. Do and T. Friščić. ACS Cent. Sci. 2017, 3, 13-19.

Keywords: antidiabetic drugs, multicomponent crystals, liquidassisted grinding 